\title{
Repositorio en la Nube de Buenas Prácticas para el Desarrollo de Competencias Transversales mediante un Aprendizaje Basado en Proyectos Arquitectónicos Guidelines Repository on the Cloud for Developing Soft Skills through a Arquitectural Projects based Learning
}

Carla Sentieri ${ }^{1}$, Damià Segrelles², carsenom@pra.upv.es,dquilis@dsic.upv.es
${ }^{2}$ Instituto de Instrumentación para Imagen Molecular (I3M)
Centro mixto CSIC - Universitat Politècnica de València
Valencia, España proceso Bolonia. El eje fundamental de estos planes, es dotar a los alumnos de un conjunto de habilidades que les capacite en aquellas competencias demandadas por el mundo empresarial, con el objeto de facilitar y acelerar a los egresados su integración plena en el mercado laboral.

En este contexto, podemos encontrar distintas formas de clasificar las competencias, sin embargo, existe una que parece poner de acuerdo a toda la comunidad universitaria, en la que se distinguen dos tipos (González, Wagenaar, 2003), las Competencias Específicas (CEs) y Transversales (CTs). El trabajo que se presenta en este artículo se centra en las CTs en el ámbito de la arquitectura, que son aquellas que definen un perfil que no se asocia a ningún área de conocimiento específico, y son transferibles a una amplia variedad de contextos laborales (Ej. trabajar en equipo, creatividad, etc...).

En arquitectura, proyectar es una forma idónea y una oportunidad para introducir en el aula Actividades Educativas (A.E) innovadoras que permitan el desarrollo y la correspondiente evaluación de las CTs. Proyectar en arquitectura es una aventura fascinante e intelectualmente enriquecedora que requiere de una labor muy creativa y compleja (Cosme, 2008). Los múltiples factores que intervienen, la diversidad de conocimientos y técnicas que debe poseer el proyectista para desarrollar esta actividad creadora con el control crítico necesario, hacen que el aprendizaje del diseño de proyectos sea una de las disciplinas más arduas con las que se tiene que enfrentar un estudiante de arquitectura (Sentieri, Castellanos, López, 2009) (de Anasagasti, 1995). Hoy en día, las escuelas de arquitectura se encargan de impartir esta disciplina, fundamentalmente a través de metodologías fundamentadas sobre un Aprendizaje Basado en Proyectos (ABPs) que se conciben a través de la ejecución de proyectos arquitectónicos de forma individual o grupal por parte de los alumnos. Los ABPs son un método de aprendizaje que definen un conjunto de actividades organizadas por etapas para la consecución de un objetivo. Dado que los proyectos son la columna vertebral de muchas de las asignaturas relacionadas con el área de arquitectura, parece idóneo utilizar y rediseñar sus actividades para el desarrollo de las CTs, dado que en los proyectos arquitectónicos, los alumnos tienen: la necesidad de diseñar y proyectar un espacio mediante un instrumental específico con programas de cálculo

\footnotetext{
${ }^{1}$ Espacio Europeo de Educación Superior. http://www.ehea.info
} 
y diseño en el desarrollo del proyecto; donde se trabaja en grupo y se requiere de liderazgo para los análisis del lugar y los antecedentes; donde se exponen públicamente los resultados; donde se desarrolla el pensamiento crítico con la participación de los alumnos en los comentarios y revisiones de los ejercicios, todo ello, dentro de un plazo determinado y bien planificado, y teniendo en cuenta que el arquitecto presta un servicio a la sociedad por lo que debe conocer los problemas contemporáneos y debe desarrollar una actitud ética, medioambiental y profesional. Por todo ello, este trabajo se centra en las metodologías de trabajo y evaluación para desarrollar competencias transversales en arquitectura.

Para desarrollar las actividades específicas de los proyectos, además de metodologías de trabajo y evaluación, es habitual el uso de herramientas de Tecnologías de la Información y Comunicación (TIC) que soportan dichas actividades (p.e. en arquitectura se utilizan herramientas software para el diseño de planos, cálculo de estructuras etc...). Esto es debido a que está demostrado que la combinación de las TIC y metodologías apropiadas mejoran significativamente lod procesos de enseñanza-aprendizaje (Campbell, Bourne, Mosterman y Brodersen, 2002) (Fraser, Pillay, Tjatindi y Case, 2007).

Una de las carencias existentes es la escasez de repositorios para reproducir y adaptar de forma aplicada las metodologías de trabajo y evaluación de los ABP en arquitectura, y de un mecanismo que proporcione aquellas herramientas TIC que se requieran para su puesta en marcha en el caso de no disponer de ellas. Por ello entendemos que una aportación y mejora al estado del arte, sería la creación de un repositorio de Buenas Prácticas para el desarrollo y Evaluación de Competencias Transversales, que además de describir la metodología y ofrecer ejemplos prácticos de cómo adaptarlas, pueda proporcionar las herramientas TIC para su puesta en marcha.

En otras áreas, sí que existen dichos repositorios, como las de la Universitat Politècnica de Cataluña UPC ${ }^{2}$, la cual es muy útil, pero sin embargo no proporciona los recursos TIC para su puesta en marcha. En este sentido, las tecnologías Cloud parecen apropiadas, dado que permiten proveer de Entornos Virtuales Computaciones (EVC) en la nube equipados del software y hardware requerido para la puesta en marcha de A.E en diferentes áreas de la ingeniería (Segrelles, Moltó, Caballer, 2015). (Segrelles, Moltó, 2016) incluida la arquitectura (Segrelles, Martinez, Castilla, Moltó, 2017).

\section{CONTEXTO}

Este trabajo se enmarca en el contexto del desarrollo de las CTs en los alumnos que cursan titulaciones relacionadas con el área de arquitectura en Universidad Politècnica de València (UPV), dentro del marco del Proyecto de Competencias Transversales de la UPV ${ }^{3}$.

El público objetivo principal son los docentes de la UPV en el área de arquitectura, que quieran innovar es sus ABPs para poder desarrollar y evaluar las CTs.

El trabajo se ha desarrollado principalmente, sobre un conjunto de proyectos que se imparten en varias asignaturas del Grado en Fundamentos de la Arquitectura (GFA), Máster

\footnotetext{
2 http://www.upc.edu/bonespractiquesdocents/2013/es/

3 Proyecto de Competencias Transversales de la UPV. http://competenciast.webs.UPV.es
}

Universitario en Arquitectura (MUARQ), Máster Universitario en Arquitectura Avanzada, Paisaje, Urbanismo y diseño (MAAPUD) y el Máster Universitario en Conservación del Patrimonio Arquitectónico (MUCP) de la Universitat Politècnica de València (UPV).

\section{A. Proyecto de Competencias Transversales en la UPV}

El Proyecto de Competencias Transversales de la UPV establece una estrategia de evaluación sistematizada de estas competencias, definiendo para cada titulación de grado y postgrado las asignaturas en las que el alumno debe adquirir, a través de actividades específicas, cada competencia y también definiendo las asignaturas donde se debe de evaluar el grado de alcance de las mismas por parte del alumno, las cuales se denominan asignaturas "punto de control”. El Proyecto de Competencias Transversales de la UPV define 13 CTs y determina tres niveles de alcance a evaluar para cada competencia: Nivel 1: Primeros cursos de grado; Nivel 2: Al finalizar el grado; Nivel 3: Al finalizar el postgrado. El objetivo final de la evaluación es la de acreditar la adquisición de las competencias en sus tres niveles, tanto individualmente -para cada estudiante-, como de forma agregada -para el conjunto de una promoción de un título- al finalizar los estudios, haciendo constar en el currículo de cada alumno dicha evaluación.

\section{B. Necesidad}

La puesta en marcha del Proyecto de Competencias Transversales de la UPV supuso en el curso 2015/2016, que todos los profesores responsables de cada asignatura diseñasen, integrasen y describiesen en las guías docentes actividades específicas para el desarrollo y evaluación de las CTs, generalmente mediante el uso y aplicación de metodologías activas de aprendizaje, dada su idoneidad, y además en el caso de las asignaturas "punto de control" también el diseño e integración de actividades de evaluación para recoger las evidencias que permitieran valorar de forma cuantitativa y/o cualitativa los indicadores que evalúan el grado de alcance de las CTs. Para facilitar la tarea de evaluación, la UPV dentro del Proyecto de Competencias Transversales ha desarrollado un conjunto de rúbricas ${ }^{4}$ que definen de forma genérica los ítems a valorar en cada competencia según el nivel a evaluar y que cada profesor responsable de las asignaturas "puntos de control" debe adaptar a su asignatura.

Una de las principales dificultades del cuerpo docente de la UPV a la hora de poner en marcha el proyecto de Competencias Transversales de esta universidad, ha sido en muchos casos, la complejidad de aplicar determinadas metodologías para el desarrollo y evaluación de CTs, dado que no se disponía de las herramientas, formación adecuada y guías que facilitasen su puesta en marcha.

\section{Objetivo}

El eje principal de este trabajo se restringe al área de arquitectura y los proyectos que allí se desarrollan, y cuyo objetivo principal es el de ofrecer al cuerpo docente un repositorio de guías de buenas prácticas en la nube que faciliten el desarrollo y evaluación de CTs de una forma metódica, reproducible y en lo posible automatizada.

\footnotetext{
${ }^{4}$ Papel de las rúbricas en la evaluación de las competencias transversales
} UPV. http://www.upv.es/entidades/ICE/info/U0702453.pdf

Octubre 4-6, 2017, Zaragoza, ESPAÑA

IV Congreso Internacional sobre Aprendizaje, Innovación y Competitividad (CINAIC 2017) 


\section{DESCRIPCIÓN}

En esta sección se describe en primer lugar las actividades realizadas para la organización del repositorio y a continuación una descripción del proceso de cómo se han realizado las actividades en un caso concreto de proyectos arquitectónicos.

\section{A. Actividades de trabajo}

Las asignaturas de proyectos arquitectónicos tienen como objetivo formar al alumno y capacitarlo para desarrollar proyectos arquitectónicos, con diferentes grados de complejidad en cada curso, mediante la realización de un proyecto arquitectónico.

La realización de un proyecto/anteproyecto arquitectónico consiste en el diseño de un edificio con un programa concreto en un lugar determinado, con el desarrollo de planos y memorias que permitan su trasmisión a otros agentes para que lo puedan ejecutar. Este trabajo, que es una de las tareas a las que se dedica mayoritariamente el arquitecto, se desarrolla dentro de las aulas de la manera más real posible para que el alumno comience a desarrollar las competencias que necesitará en su ejercicio profesional.

Desde el Año 2010, el grupo de Innovación y calidad en el proyecto arquitectónico (ICAPA), en el que se encuentran los autores, ha ejecutado diferentes Proyectos de Innovación y Mejora Educativa (PIME), cuyos esfuerzos se han centrado principalmente en adaptar las asignaturas de Proyectos al Espacio Europeo de Educación Superior (EEES) (Domingo, Sentieri, 2011) y crear herramientas en la nube para el desarrollo y evaluación de competencias transversales en diferentes ámbitos (Segrelles et al. 2015). (Segrelles et al., 2016), entre los que se encuentra el área de arquitectura (Segrelles et al, 2017).

Los miembros de ICAPA han participado en los grupos de trabajo de la UPV que han desarrollado diferentes rúbricas para la evaluación de las competencias transversales: "Diseño y Proyecto”, “Comunicación Efectiva”, “Trabajo en grupo y liderazgo" y "Pensamiento crítico".

En paralelo, otras experiencias de recopilación de buenas prácticas han sido estudiadas por miembros del equipo, como se ha indicado anteriormente. Este tipo de información, es útil, pero la necesidad de hacer una herramienta más visual e intuitiva, tal y como sucede con las páginas de recetas de cocina, fue el origen de la innovación. Por lo tanto, se procedió a maquetar de forma provisional y como punto de partida, durante el curso 2016/2017, la futura plataforma ${ }^{5}$ :

\section{B. Describiendo procesos ABP con cada una de las A.Es $y$ trabajando las competencias}

Cada uno de los procesos de ABP conlleva el desarrollo de actividades que en algunas ocasiones se fragmentan haciéndolas más explicitas y en otras se van desarrollando en una continua iteración cíclica con todos los temas a la vez. Con el objetivo de clarificar y poder evaluar cada una de las partes del proceso, se procedió a analizar y descomponer el proceso del proyecto. El desarrollo de un proyecto arquitectónico no es lineal, su complejidad radica en el manejo

\footnotetext{
${ }^{5}$ Maqueta Guía de Buenas Prácticas.

https://competenciastransversalesupv.wordpress.com/portfolio/ciudad
} es-invisbles de muchas variables al mismo tiempo, y el hecho de descomponerlas ayuda a tomar conciencia de la existencia de cada factor, pero sin olvidar que es un proceso global. Una vez determinadas las actividades en las que se puede descomponer el proceso de desarrollo de los proyectos realizados en los diversos cursos, se establecieron categorías en función de la fase del proyecto que trabajan: análisis del lugar, el análisis del programa/usuario, análisis de referentes, primeras ideas o planteamientos y propuesta final.

Para cada una de las actividades se describieron las competencias que trabajaban, o podían trabajar, durante el desarrollo y se seleccionó una rúbrica de evaluación del nivel I, II o III de las rúbricas desarrolladas en el proyecto de competencias transversales de la UPV, para cada competencia en función del curso del alumnado. La existencia de una rúbrica común permite comparar resultados sobre el desarrollo de cada competencia y dado que el profesorado que compone el grupo pertenece a niveles/cursos distintos constatar la evolución y la validez del proceso.

Todo este material de actividades para el desarrollo del proyecto y el trabajo y evaluación de las CTs está disponible en el propio repositorio.

\section{RESUltados}

Tabla 1. Competencias Transversales por Actividad Educativa.

\begin{tabular}{|l|l|}
\hline Actividades & Competencias Transversales \\
\hline Educativas & \\
\hline lugálisis del & -Trabajo en grupo y liderazgo. \\
& -Comunicación efectiva oral y gráfica. \\
& -Planificación y gestión del tiempo. \\
& -Pensamiento crítico. \\
& -Conocimiento de problemas contemporáneos. \\
& -Responsabilidad ética, medioambiental y profesional. \\
& -Instrumental específica. \\
\hline Análisis del & -Trabajo en grupo y liderazgo. \\
programa & -Comunicación efectiva oral y gráfica. \\
& -Planificación y gestión del tiempo. \\
& -Pensamiento crítico. \\
& -Conocimiento de problemas contemporáneos. \\
& -Responsabilidad ética Medioambiental y profesional. \\
& -Instrumental específica. \\
\hline Análisis & -Comunicación efectiva oral y gráfica. \\
referentes & -Planificación y gestión del tiempo. \\
vivienda & -Pensamiento crítico. \\
& -Conocimiento de problemas contemporáneos. \\
& -Responsabilidad ética, medioambiental y profesional. \\
& -Instrumental específica. \\
\hline Presentación & -Diseño y proyecto. \\
\hline $\begin{array}{l}\text { Primeros } \\
\text { planteamientos }\end{array}$ & -Comunicación efectiva oral y gráfica. \\
y desarrollo & -Planificación y gestión del tiempo. \\
& -Pensamiento crítico. \\
& -Conocimiento de problemas contemporáneos. \\
& -Responsabilidad ética medioambiental y profesional \\
& -Instrumental específica. \\
\hline & -Comunicación efectiva oral y gráfica \\
& -Planificación y gestión del tiempo \\
& -Pensamiento crítico \\
& -Conocimiento de problemas contemporáneos \\
& -Responsabilidad ética, medioambiental y profesional \\
& \\
\hline
\end{tabular}

En esta sección se muestra primero y a modo de ejemplo, las partes del desarrollo de un proyecto arquitectónico concreto, y como esta ha sido descompuesto en actividades específicas. A continuación, se muestra la apariencia de estas A.Es en el repositorio. 


\section{A. Ejemplo de A.Es de un Proceso ABP}

En este ejemplo, se desarrolla un proyecto de alojamiento colectivo con una pequeña escuela infantil enunciando cada una de las A.Es junto con las competencias transversales que se trabajan (Tabla 1).

\section{Análisis del Lugar}

Consiste en un análisis del lugar desde el punto de vista geográfico, histórico, socio-cultural y urbanístico/arquitectónico. Los alumnos, organizados en grupos de cinco, entregan un pdf con el análisis y este se expone públicamente. (Figura 1).

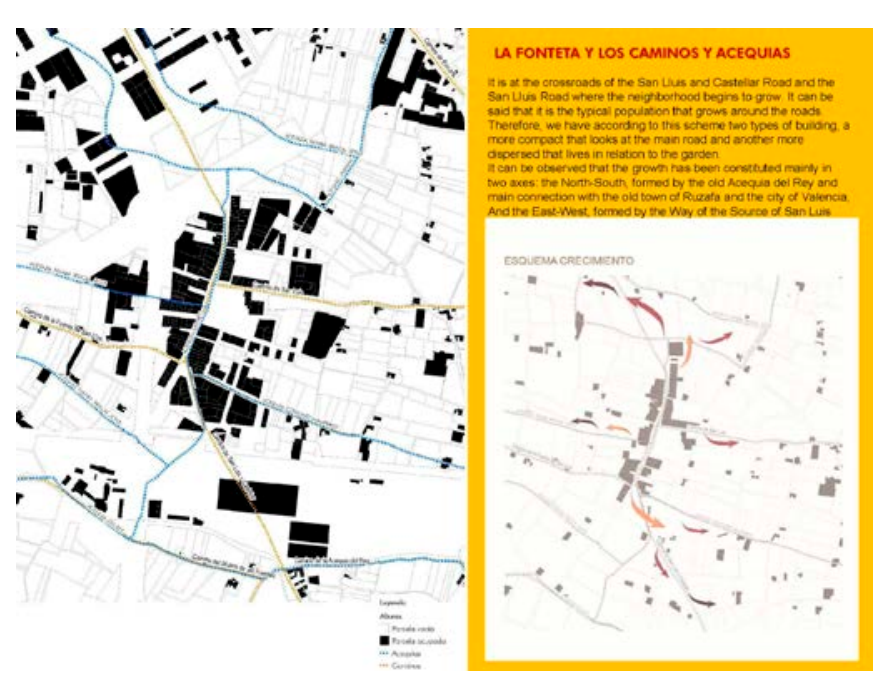

Figura 1. Entregable "Análisis del lugar”. Proyectos 2. Taller 4. ETSA UPV. Curso 2016-17.

\section{Análisis del programa/usuarios}

Consiste en analizar, revisar el programa propuesto o el conveniente para el proyecto solicitado, desde el punto de vista de los usuarios: necesidades funcionales, espaciales, emocionales (Figura 2).
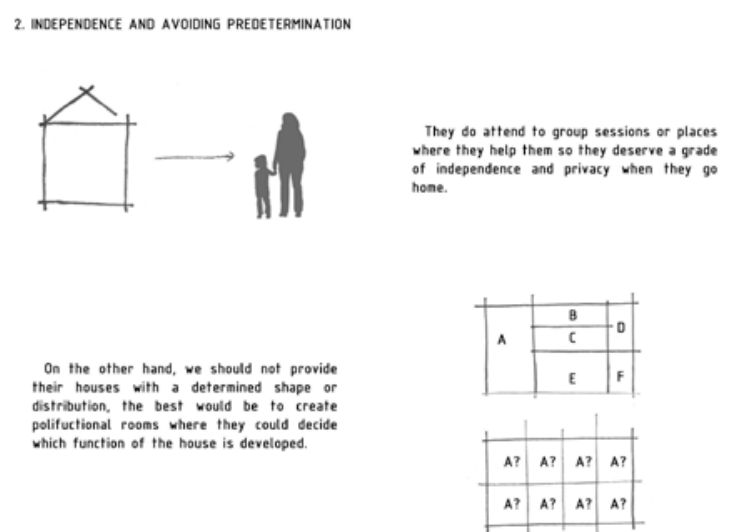

Figura 2. Entregable “Análisis de usuarios”. Alumno: Carlos Silvestre. Proyectos 2. Taller 4. ETSA UPV. Curso 2016-17.

\section{Análisis de proyectos de vivienda colectiva.}

Consiste en analizar proyectos de vivienda desde la funcionalidad, la estructura, la construcción, la materialidad (Figura 3).
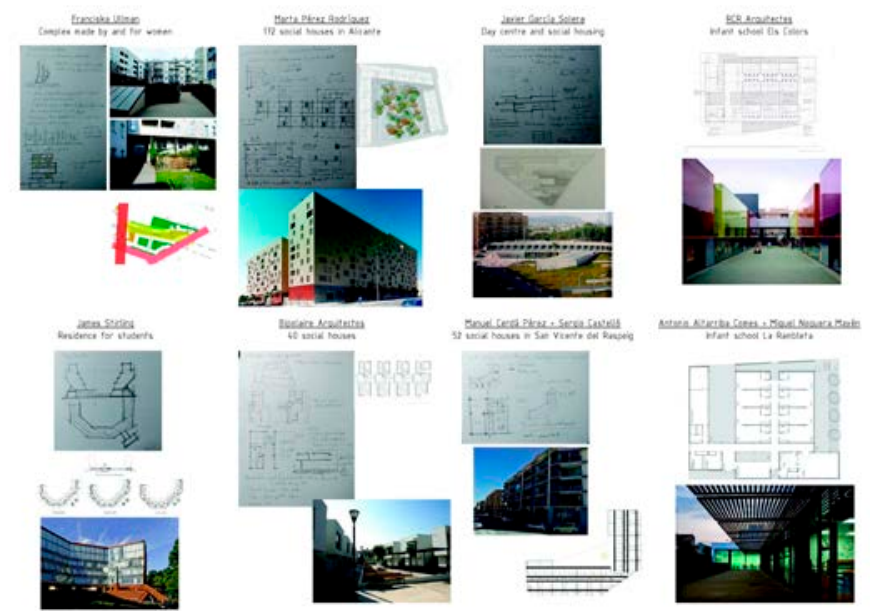

Figura 3. Entregable "Análisis de proyectos de vivienda colectiva”. Alumno: Carlos Silvestre. Proyectos 2. Taller 4. ETSA UPV. Curso 2016-17.

\section{Primeras planteamientos y desarrollo}

Los alumnos de forma individual proponen las primeras ideas o aproximaciones a la solución del proyecto (Figura 4).

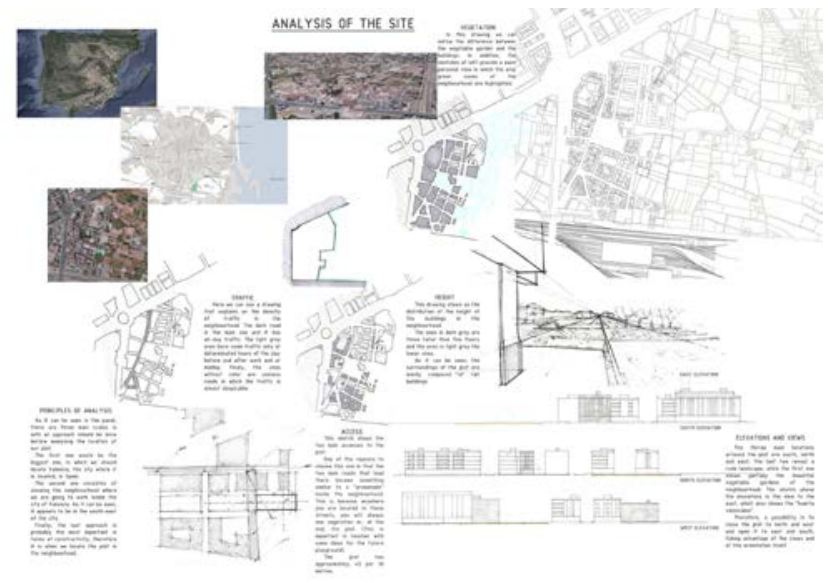

Figura 4. Entregable "Primeras ideas". Alumno: Carlos Silvestre. Proyectos 2. Taller 4. ETSA UPV. Curso 2016-17.

Durante el desarrollo del proyecto y de forma continua, la mitad de cada una de las sesiones de clase/taller se utiliza para la revisión del proyecto de cada alumno en sesiones públicas donde el alumno expone el desarrollo de su proyecto.

\section{Presentación final y entrega.}

En la fecha indicada en el calendario como entrega final, el alumno presenta todo el documento correspondiente al desarrollo del proyecto con planos y memoria, una maqueta del proyecto y un panel resumen formato DIN A1 con toda la información más relevante. (Figura 5). 


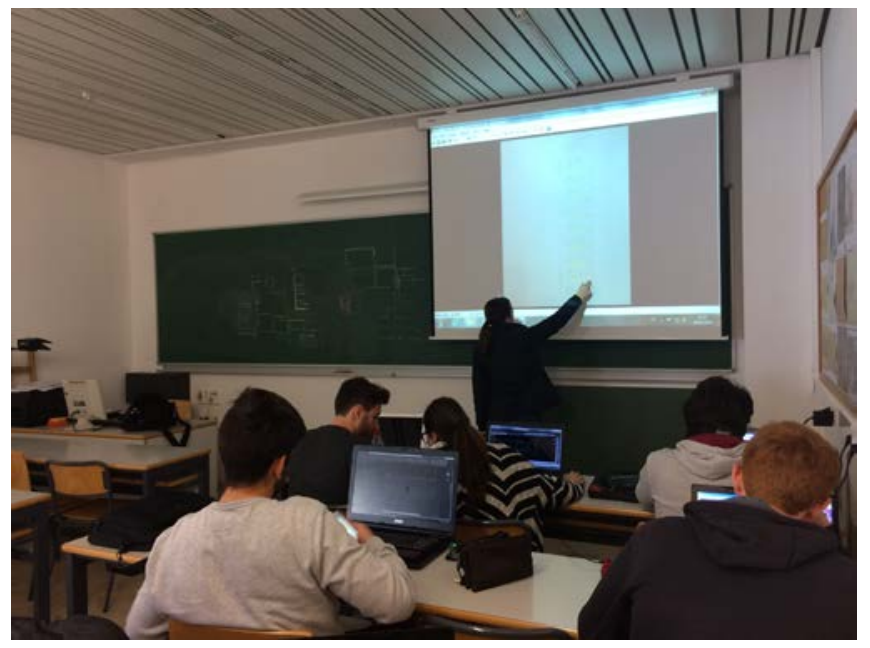

Figura 5. Exposición del trabajo de desarrollo en el aula. Proyectos 2. Taller 4. ETSA UPV. Curso 2016-17

\section{B. Implementación en el repositorio}

Todas estas actividades se recogen en un repositorio en el que en la primera pantalla de presentación se puede hacer una selección de A.Es por competencia transversal trabajada o por tipo de A.E. En la parte inferior aparece un link que enlaza con la página institucional del Proyecto de Competencias UPV ofreciendo videos explicativos para cada competencia e información sobre su desarrollo. (Figura 6 y 7).

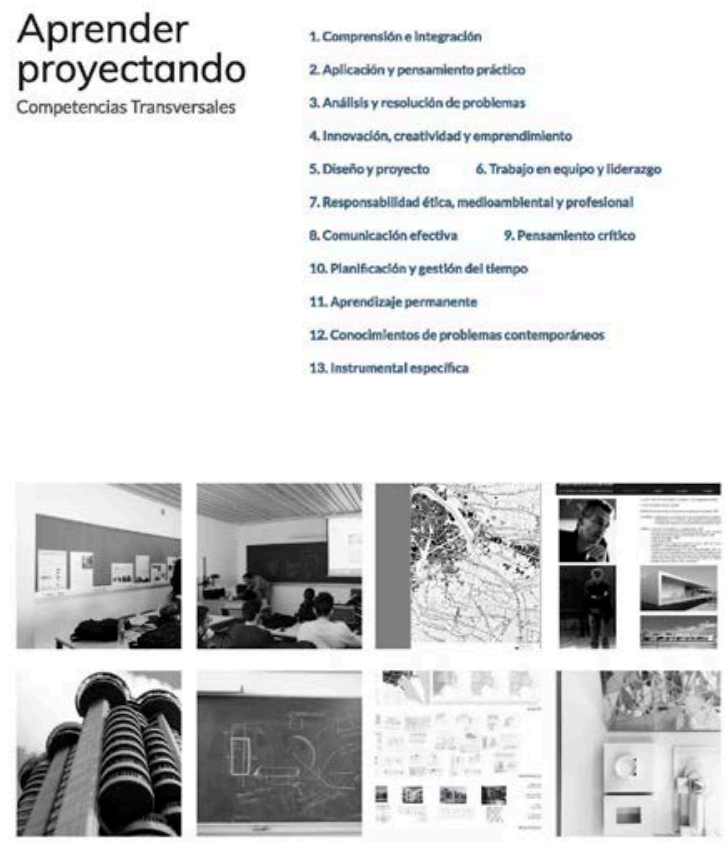

Figura 6. Portada del repositorio ${ }^{6}$ señalando el link a las competencias.

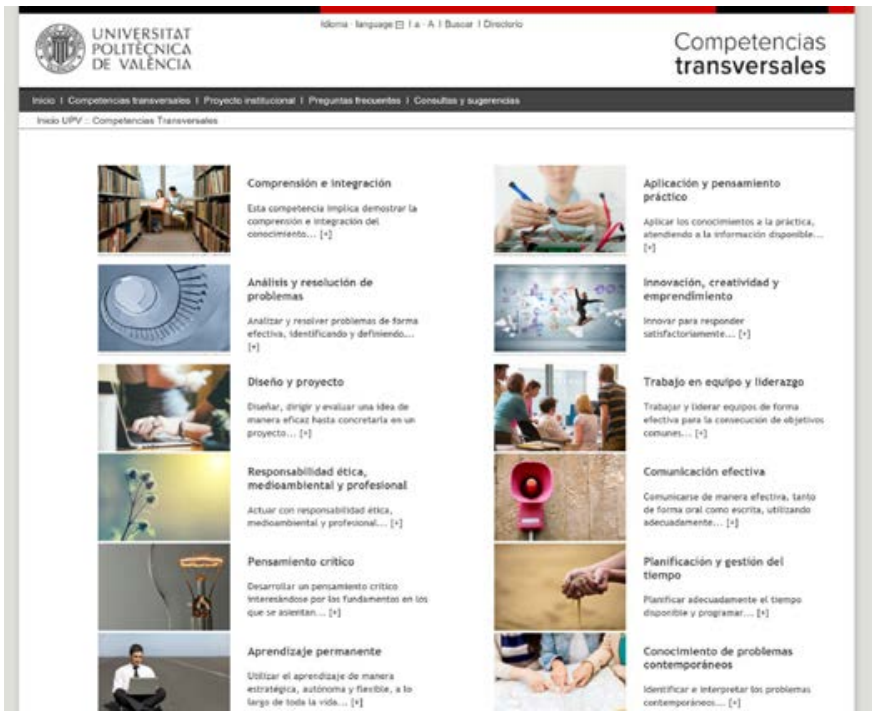

Figura 7. Página institucional ${ }^{7}$ del Proyecto de Competencias Transversales UPV con los enlaces a videos.

Cada A.E se describe de forma precisa, escueta, a modo de receta con los siguientes apartados: datos, diseño de la actividad describiendo los ingredientes o necesidades de partida, la elaboración- el proceso y la evaluación junto con las rúbricas empleadas para la valoración de las competencias transversales. (Figura 8 y 9).

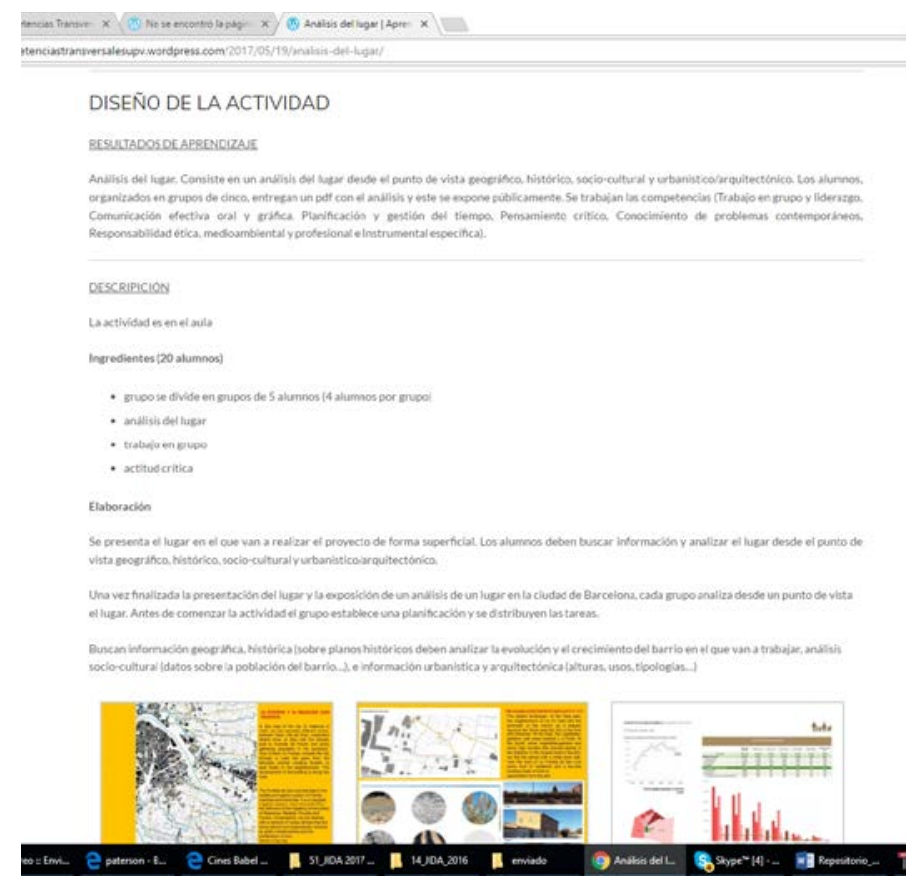

Figura 8. Captura de pantalla ${ }^{8}$, Diseño de la actividad “Análisis del lugar".

${ }^{6}$ https://competenciastransversalesupv.wordpress.com/

${ }^{7}$ http://www.upv.es/contenidos/COMPTRAN/

${ }^{8}$ https://competenciastransversalesupv.wordpress.com/2017/05/19/analisisdel-lugar/

Octubre 4-6, 2017, Zaragoza, ESPAÑA

IV Congreso Internacional sobre Aprendizaje, Innovación y Competitividad (CINAIC 2017) 


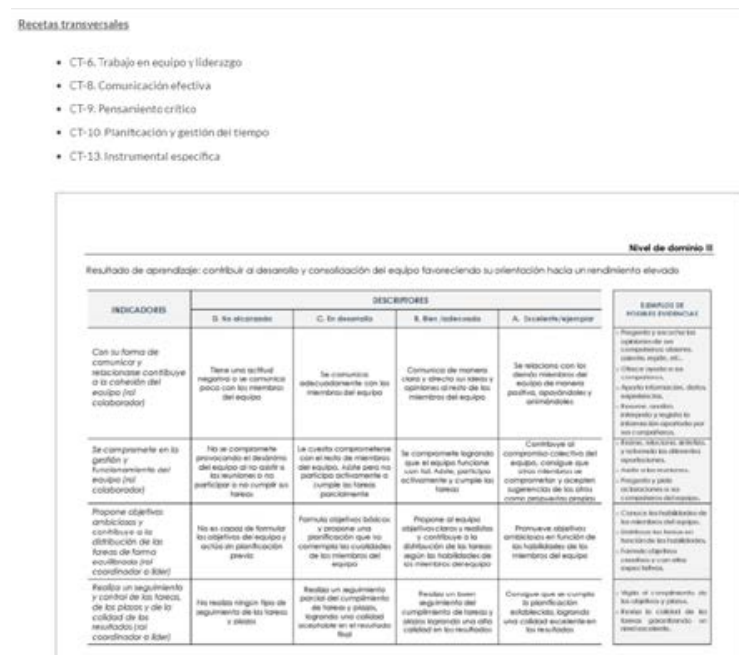

Figura 9. Captura de pantalla ${ }^{9}$ rúbrica de evaluación de competencia transversal: Trabajo en equipo y liderazgo.

\section{CONCLUSIONES}

El repositorio generado permite la utilización de las guías por parte de cualquier profesor que trabaje CTs, en arquitectura particularmente, pero también en otras disciplinas que trabajen por ABP. El hecho de describir con imágenes el contexto, el desarrollo y la evaluación permite que cada profesor se plantee nuevas actividades vinculadas con su materia específica, aprovechando las rúbricas para las competencias transversales de las que se disponen, y la experiencia en tiempos para las planificaciones. Es fácilmente sostenible porque solo requiere tomar datos de las actividades que se van realizando cada año, para ir ampliando el repositorio, incluso se podría plantear como trabajo futuro algún tipo de blog/web tipo participativo donde cada uno subiera o volcara su información, de tal modo que se generase una red de buenas prácticas.

Además el propio repositorio, al proporcionar los recursos en la nube, permite embeber todo el material en la propia plataforma, permitiendo el despliegue en la nube de todos los recursos que se requieren para la puesta en marcha de las diferentes actividades. Si bien es cierto, queda como trabajo futuro embeber en todas las guías publicadas los recursos, dado que actualmente solo se proporciona para el ámbito de trabajos cooperativos arquitectónicos (Segrelles et al, 2017).

Como se ha podido observar el trabajo de las competencias debe ser continuo a lo largo del curso y se producen numerosas revisiones que en muchas ocasiones no se registran porque se hacen de forma oral.

Para mejorar el trabajo de las competencias se debe establecer un protocolo más específico y evaluar cada una de ellas en momentos denominados "puntos de control", pero sin perder de vista que el objetivo es el formativo y no el "clasificatorio". El tiempo que conlleva registrar todos estos datos puede hacer que se pierda un tiempo muy valioso para nuevas revisiones o feed-back.

\footnotetext{
${ }^{9}$ https://competenciastransversalesupv.wordpress.com/2017/05/19/analisisdel-lugar/
}

Hasta ahora el material utilizado son imágenes, pero tras la participación en la elaboración de los videos institucionales del proyecto Competencias Transversales UPV, el siguiente objetivo será documentar todas estas actividades con videos de 2 minutos de tal forma que su comprensión sea más rápida.

\section{AGRADECIMIENTOS}

En este trabajo, los autores agradecen por la financiación recibida del Vicerrectorado de Estudios, Calidad y Acreditación de la Universitat Politècnica de València para desarrollar el Proyecto de Innovación y Mejora Educativa (PIME) "Entornos Virtuales Computacionales para la Evaluación de Competencias Transversales en la Nube”, con referencia A04.

\section{REFERENCIAS}

Campbell, J. O., Bourne, J. R., Mosterman, P. J., \& Brodersen, A. J. (2002). The effectiveness of learning simulations for electronic laboratories. Journal of Engineering Education, 91(1), 81-87.

Cosme, A. M. (2008). El proyecto de arquitectura: concepto, proceso y representación (Vol. 16). Barcelona, España.

De Anasagasti, T. (1995). Enseñanza de la arquitectura: cultura moderna técnico artística (Vol. 2). Madrid, España: Instituto Juan de Herrera.

Domingo, D., \& Sentieri, C. (2011). Blended learning in the area of the architectural project. An experience of adjustment to the European space for higher education in the school of architecture of Valencia. In EDULEARN11 Proceedings (pp. 3076-3082). IATE

Fraser, D. M., Pillay, R., Tjatindi, L., \& Case, J. M. (2007). Enhancing the learning of fluid mechanics using computer simulations. Journal of Engineering Education, 96(4), 381

González, J., \& Wagenaar, R. (Eds.). (2003). Tuning educational structures in Europe. Final report. Phase one. Bilbao: University of Deusto.

Segrelles, J. D., \& Moltó, G. (2016). Assessment of cloudbased Computational Environments for higher education. In Frontiers in Education Conference (FIE), 2016 IEEE (pp. 1-9). IEEE.

Segrelles, J. D., Molt, G., \& Caballer, M. (Noviembre de 2015). Remote Computational Labs for Educational Activities via a Cloud Computing Platform. In 2015 Proceedings of the Information Systems Education Conference (ISECON) (pp. 309-321).

Segrelles, J. D., Martinez, A., Castilla, N., \& Moltó, G. (2017). Virtualized Computational Environments on the cloud to foster group skills through PBL: A case study in architecture. Computers \& Education, 108, 131-144.

Sentieri, C., Castellanos, R., \& López, R. (Noviembre de 2009). El aprendizaje de proyectos centrado en la indagación-comparación. Actas III Jornadas Internacionales UPM sobre Innovación Educativa y Convergencia Europea (INECE 09), Madrid, España. 